\title{
Inhibition of Insulin-Like Growth Factor-1-Induced Sebum Production by Bilobetin in Cultured Human Sebocytes
}

\author{
Cong Wang ${ }^{1,2}$, Yul-Lye Hwang ${ }^{2}$, Xue Mei Li ${ }^{2,3}$, Soo Jung Kim², Ming Ji Zhu ${ }^{1}$, Jeung-Hoon Lee ${ }^{2,3,4}$, \\ Ri-Hua Jiang ${ }^{1}$, Chang Deok Kim ${ }^{2,3}$ \\ ${ }^{1}$ Department of Dermatology, China-Japan Union Hospital of Jilin University, Changchun, China, Departments of ${ }^{2}$ Dermatology and \\ ${ }^{3}$ Medical Science, Chungnam National University School of Medicine, ${ }^{4}$ Skin Med Company, Daejeon, Korea
}

\begin{abstract}
Background: Sebocytes are the major cells of sebaceous gland. The essential role of sebocytes is the production of sebum, a specific lipid mixture, that covers the body surface and provides the barrier function. At puberty, sebum production increases under the effects of various stimuli including androgens and insulin-like growth factor-1 (IGF-1). Excessive sebum production changes the microenvironment surrounding hair follicle, often leading to the onset of acne. Objective: We previously performed screening test using cultured human sebocytes, and found that bilobetin had a potential for inhibiting lipid production. The aim of this study is to demonstrate the effects of bilobetin on IGF-1-induced lipogenesis in sebocytes. Methods: We pretreated simian virus $40 \mathrm{~T}$ (SV40T)-transformed sebocytes with bilobetin then stimulated with IGF-1. Effects of bilobetin on lipogenesis of sebocytes were examined by thin layer chromatography and Western blot. Results: Bilobetin markedly inhibited IGF-1induced lipid production in sebocytes, especially in terms of
\end{abstract}

Received August 30, 2018, Revised November 29, 2018, Accepted for publication November 29, 2018

Corresponding author: Chang Deok Kim, Department of Dermatology, Chungnam National University School of Medicine, 266 Munhwa-ro, Jung-gu, Daejeon 35015, Korea. Tel: 82-42-580-6704, Fax: 82-42-280-8459, E-mail: cdkimd@cnu.ac.kr

ORCID: https://orcid.org/0000-0001-9341-6491

Ri-Hua Jiang, Department of Dermatology, China-Japan Union Hospital of Jilin University, Changchun, Jilin 130033, China. Tel: 86-431-84995523, Fax: 86-431-84641026, E-mail: jrh1963@163.com ORCID: https://orcid.org/0000-0002-7957-9427

This is an Open Access article distributed under the terms of the Creative Commons Attribution Non-Commercial License (http://creativecommons. org/licenses/by-nc/4.0) which permits unrestricted non-commercial use, distribution, and reproduction in any medium, provided the original work is properly cited.

Copyright $\odot$ The Korean Dermatological Association and The Korean Society for Investigative Dermatology production of squalene and wax ester. Supporting these results, bilobetin showed significant inhibitory effect on squalene synthase promoter activity. In addition, bilobetin significantly down-regulated lipogenic transcription factors such as sterol response element binding protein (SREBP)-1 and SREBP-2. To delineate the possible action mechanism, we investigated the effect of bilobetin on intracellular signaling. As a result, bilobetin inhibited IGF-1-induced phosphorylation of AKT. Conclusion: Together, these results suggest that bilobetin has an inhibitory potential on sebum production in sebocytes, being applicable for acne treatment.

(Ann Dermatol 31(3) $294 \sim 299,2019$ )

\section{-Keywords-}

Acne, Bilobetin, Insulin-like growth factor I, Sebocytes

\section{INTRODUCTION}

The primary role of sebocytes is the making sebum, the specialized lipid complex, that covers the surface of skin thereby providing a barrier function ${ }^{1}$. Sebum production is controlled by various factors such as androgens, corticotropin-releasing hormone, vitamin $\mathrm{D}$ and insulin-like growth factor-1 (IGF-1) $)^{2}$. At puberty, sebum production increases in accordance with the increase of circulating androgens, and the resultant sebum increase often leads to the development of acne. When sebocytes produce excess sebum, the microenvironment surrounding hair follicle changes into lipid-rich hypoxic condition that enables the flourishing of lipidophilic commensal bacteria such as Propionibacterium acnes. In addition, the metabolites produced by microbes trigger the activation of perifollicular inflammation, exacerbating the acne condition ${ }^{3,4}$. 
Currently, many drugs are employed for treatment of acne. For example, the antibiotics such as tetracycline, doxycycline and minocycline are used for controlling microbes. In addition, vitamin A derivatives such as adapalene, isotretinoin and tazarotene are used for inhibiting the hyperkeratinization of pilosebaceous duct ${ }^{5}$. Although many drugs are widely used for treatment of acne, there is still a demand for the development of new treatments that have little adverse effects and high efficacy. Since the excess production of sebum is the starting event in pathogenesis of acne, targeting the sebum production of sebocytes is a potential strategy for acne therapy.

In a previous screening test using cultured human sebocytes, we found that bilobetin had strong anti-lipogenic potential. In this study, we demonstrate that bilobetin inhibits IGF-1-induced sebum production, suggesting that bilobetin can be developed for acne treatment.

\section{MATERIALS AND METHODS}

\section{Ethics statement}

Skin tissues were obtained from volunteers under the written informed consent, and the present study was approved from the ethical committee of the Institutional Review Board of Chungnam National University Hospital (IRB no. 1011-135, 2016-07-009).

\section{Cell culture}

Human scalp tissues were immersed briefly in $70 \%$ ethanol for sterilization, then sebaceous glands were separated using microforcep under the stereomicroscope. Isolated sebaceous glands were attached to the culture dish, and incubated in Sebomed ${ }^{\mathbb{R}}$ medium (Biochrom, Berlin, Germany) supplemented with $10 \%$ fetal bovine serum and 5 $\mathrm{ng} / \mathrm{ml}$ recombinant human epidermal growth factor (Life Technologies Corporation, Grand Island, NY, USA). Usually, sebocytes were grown out from explanted sebaceous glands in 1 week.

For a long and continuous maintenance of sebocytes cell line, we immortalized the primary cultured cells using a recombinant retrovirus expressing simian virus $40 \mathrm{~T}$ (SV40T) antigen. The retroviral vector pLXIN-SV40T was transfected into the retrovirus packaging cell line PT67 (Clontech Laboratories, Mountain View, CA, USA). The medium containing retrovirus was harvested, filtrated through a $0.22-\mu \mathrm{m}$ low protein binding filter (Merck KGaA, Darmstadt, Germany), and then transferred to primary cultured sebocytes. After incubation overnight, the retroviruscontaining medium was removed and fresh medium was added. The immortalized sebocytes were selected in medium containing $200 \mu \mathrm{g} / \mathrm{ml}$ of G418 (Sigma, St. Louis,
MO, USA) for 4 weeks.

\section{Cell viability test}

SV40T-transformed sebocytes (SV-sebocytes) were seeded in 6-well plate at a density of $2 \times 10^{5}$ cells/well, treated with bilobetin (Biopurify Phytochemicals Ltd., Chengdu, China) for 1 day. Cell viability was measured by MTT (3(4,5-dimethylthiazol-2-yl)-2,5-diphenyltetrazolium bromide) assay.

\section{Cell growth assay}

To measure the cell growth, $\left[{ }^{3} \mathrm{H}\right]$ thymidine uptake assay was carried out. SV-sebocytes were seeded in 6-well plate and treated with bilobetin for 2 days in the presence of 1 $\mu \mathrm{Ci}\left[{ }^{3} \mathrm{H}\right]$ thymidine (Amersham, UK). Cells were lysed with $0.1 \mathrm{~N} \mathrm{NaOH}$, then radioactivity was measured using a liquid scintillation counter.

\section{Oil Red O staining}

SV-sebocytes were grown on cover glass and treated with bilobetin. After fixation with $10 \%$ formalin for 5 minutes, cells were stained with Oil Red O solution. Stained cells were vigorously washed with distilled water, and then counterstained with hematoxylin.

\section{Lipogenesis assay}

To check the lipogenesis, a well-established ${ }^{14} \mathrm{C}$-acetate incorporation assay was used ${ }^{6}$. SV-sebocytes were treated with bilobetin for 1 day, then $2 \mu \mathrm{Ci}$ of $\left[1-{ }^{14} \mathrm{C}\right]$ acetic acid (PerkinElmer, Boston, MA, USA) was added and incubated for a further 6 hours. Cellular lipids were extracted with the solvent comprising of chloroform and methanol (2:1). After evaporation of solvent, cellular lipids were dissolved in chloroform and separated using a thin layer chromatography (TLC silica gel $60 \mathrm{~F}_{254}$; Merck KGaA). The developing buffer for TLC consists of hexane and ethyl acetate (6:1). Lipids were visualized by autoradiography.

\section{Luciferase reporter assay}

The squalene synthase-luc reporter adenovirus (Ad/SS-luc) was produced previously ${ }^{7}$. For luciferase reporter assay, SV-sebocytes were seeded in 12-well plate and infected with Ad/SS-luc for 6 hours. After removal of adenoviruscontaining medium, cells received fresh medium containing IGF-1 and bilobetin for 1 day. Luciferase activity was measured using the dual luciferase reporter assay system (Promega, Madison, WI, USA).

\section{Western blot}

Cellular extracts were prepared using a lysis buffer (Intron, Daejeon, Korea). After sodium dodecyl sulfate-polyacry- 
lamide gel electrophoresis, proteins were transferred to nitrocellulose membranes. The membranes were incubated with primary antibody, and then sequentially incubated with peroxidase-conjugated secondary antibodies. The Western bands were obtained by enhanced chemiluminescence (Intron), and quantified using ImageJ software (ver. 1.50i). The following primary antibodies were used: sterol response element binding protein-1 (SREBP-1), actin (Santa Cruz, CA, USA); SREBP-2, squalene synthase (farnesyl-diphosphate farnesyltransferase 1, FDFT1) (Abcam, Cambridge, UK); stearoyl-CoA desaturase (SCD) (Thermo Scientific, Rockford, IL, USA); IGF-1-R, phospho-IGF-1-R, AKT, phospho-AKT, ERK1/2, phospho-ERK1/2 (Cell Signaling Technology, Beverly, MA, USA).

\section{RESULTS}

Bilobetin is a biflavonoid isolated from crude leaf extracts of ginkgo biloba ${ }^{8}$. It is made up of two structurally very similar but not identical flavonoid molecules by covalent bonding (Fig. 1A). To determine the cytotoxic effect, we treated SV-sebocytes with bilobetin at various concentrations. As a result, bilobetin did not show significant cytotoxicity up to the dose of $4 \mu \mathrm{M}$ (Fig. 1B).

To investigate the effect of bilobetin on lipid production of sebocytes, we employed a well-established IGF-1-induced lipogenesis model ${ }^{6,9}$. Since IGF-1 stimulates sebocyte proliferation and lipogenesis ${ }^{10}$, we first examined the effect of bilobetin on cell growth using $\left[{ }^{3} \mathrm{H}\right]$ tymidine uptake assay. Treatment of SV-sebocytes with IGF-1 resulted in increase of cell growth, and pretreatment with bilobetin inhibited IGF-1-induced cell growth significantly (Fig. 2A). We next examined the effect of bilobetin on the production of neutral lipids by Oil Red O staining. IGF-1 treatment increased lipid production, and pretreatment with bilobetin resulted in significant inhibition of IGF-1-induced lipid production (Fig. 2B). To further analyze the lipid production of sebocytes, we performed TLC. Result showed that bilobetin markedly inhibited IGF-1-induced lipid production, including squalene and wax ester. In addition, bilobetin also blocked significantly the production of triglyceride and cholesterol (Fig. 2C). It has been well known that squalene and wax ester are the unique lipids found in sebum $^{2}$, thus we examined whether bilobetin can affect the promoter activity of squalene synthase (FDFT1) gene. Cells were transduced with recombinant adenovirus harboring the squalene synthase promoter-luc cassette, then treated with IGF-1 and bilobetin. Consistent with data obtained from TLC, IGF-1 increased the squalene synthase promoter activity, and bilobetin significantly inhibited IGF-1induced promoter activity (Fig. 2D). These results suggest that bilobetin may be applicable for specific inhibition of sebum production in sebocytes.

During the synthesis of lipids, various transcription factors and lipogenic enzymes are involved ${ }^{11,12}$. We thus investigated whether bilobetin affected the expression of several lipogenic regulators. As expected, IGF-1 treatment led to increase of lipogenic transcription factors, SREBP-1 and SREBP-2, together with increase of lipogenic enzymes such as squalene synthase (FDFT1) and SCD. Pretreatment of bilobetin significantly inhibited IGF-1-induced up-regulation of lipogenic regulators (Fig. 3A). Since it has been demonstrated that IGF-1 induces SREBP-1 via the IGF-1 receptor (IGF-1-R) and downstream signaling pathway in se-

A

B

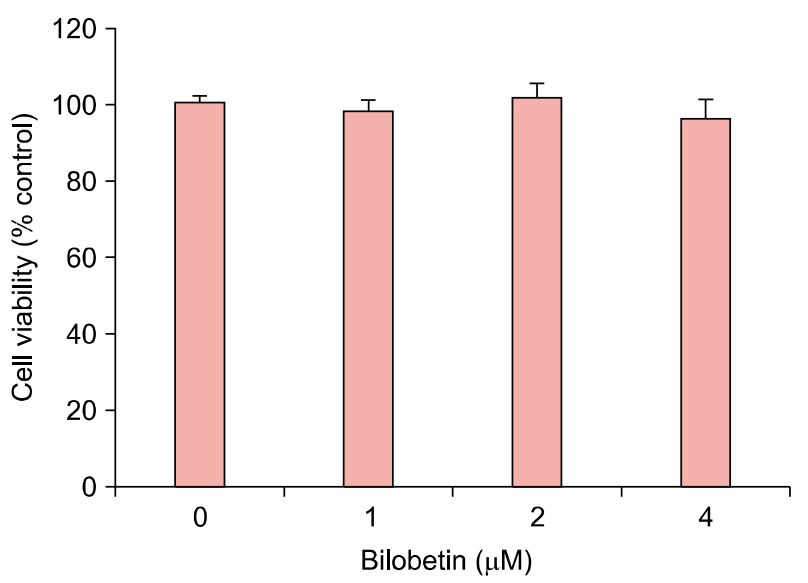

Fig. 1. (A) Structure of bilobetin. (B) Cytotoxicity of bilobetin. Simian virus $40 \mathrm{~T}$ (SV40T)-transformed sebocytes were treated with bilobetin at the indicated concentrations for 1 day. Cell viability was measured by MTT assay. The mean values \pm standard deviation are averages of triplicate measurements. 
A

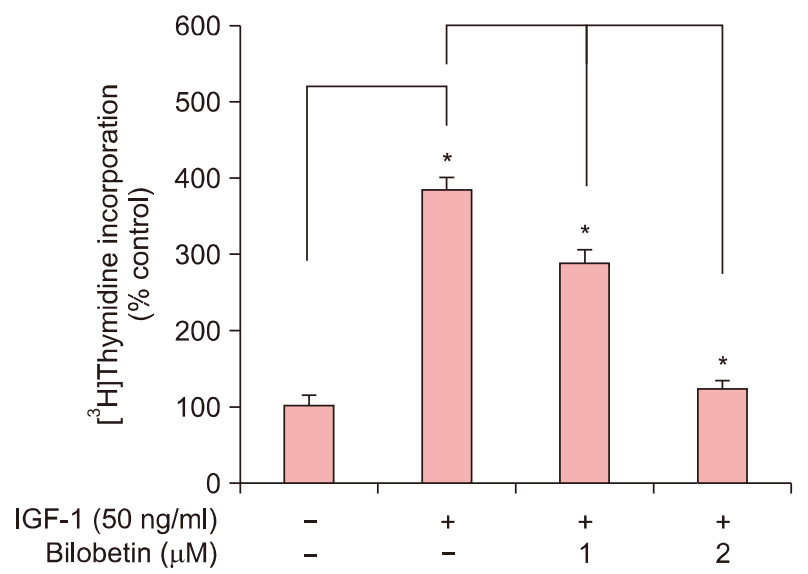

C

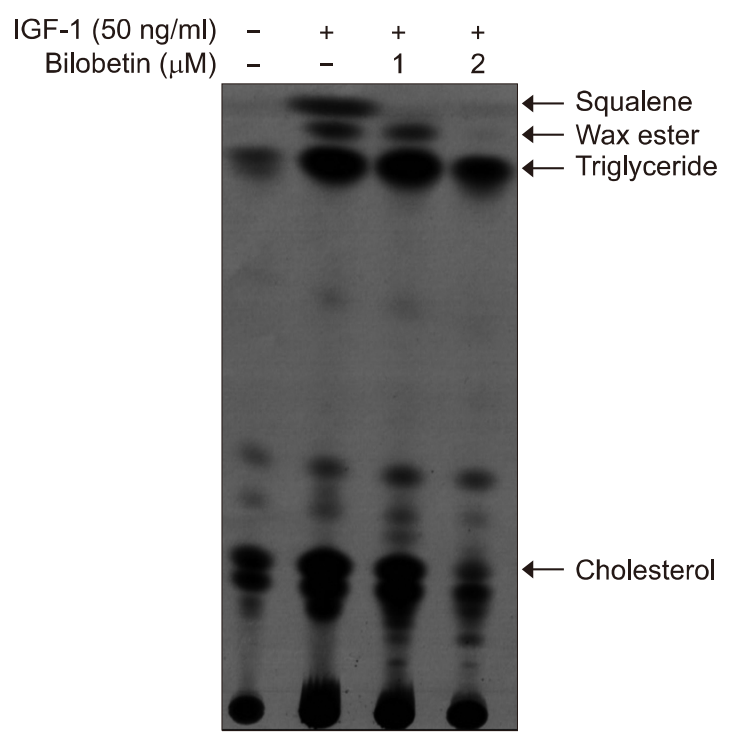

B

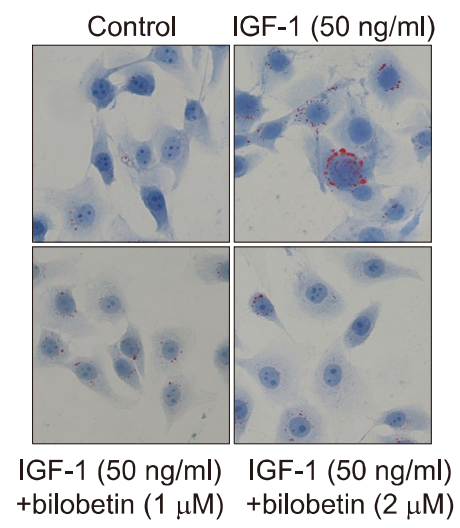

D

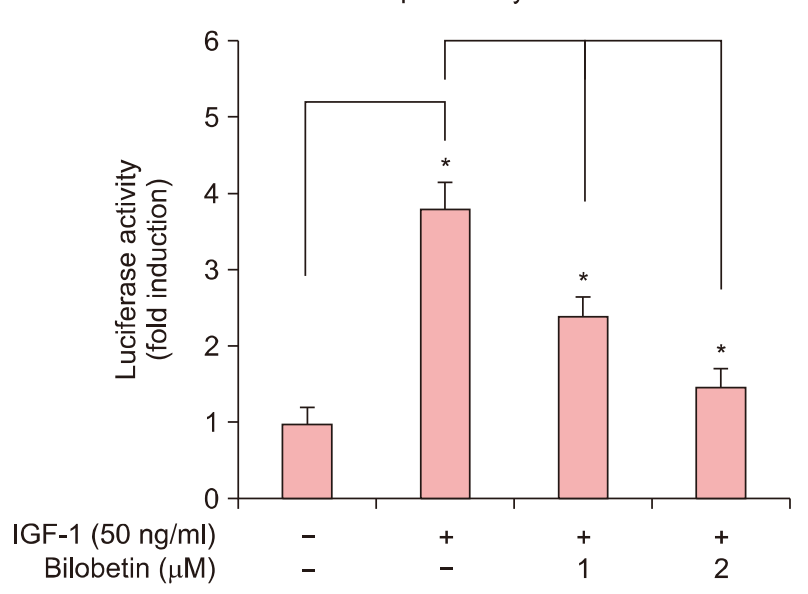

Fig. 2. (A) Effect of bilobetin on the growth of sebocytes. Simian virus $40 \mathrm{~T}$ (SV40T)-transformed sebocytes were incubated in plain media without fetal bovine serum, then treated with insulin-like growth factor-1 (IGF-1) and bilobetin for 2 days in the presence of $\left.1 \mu \mathrm{Ci}{ }^{3} \mathrm{H}\right]$ thymidine. After scintillation counting, data are expressed as percent control. The mean values \pm standard deviation (SD) are averages of triplicate measurements. Bilobetin inhibited IGF-1-induced cell growth. (B) Cells were treated with IGF-1 and bilobetin at the indicated concentrations for 1 day. Neutral lipids were detected by Oil Red $O$ staining $(\times 400)$. (C) Effect of bilobetin on IGF-1-induced lipid production was determined by thin layer chromatography. Production of squalene and wax ester was significantly inhibited by bilobetin. (D) Effect of bilobetin on the squalene synthase promoter activity. Cells were transduced with 1 multiplicity of infection of squalene synthase-luc reporter adenovirus, then treated with IGF-1 and bilobetin for 1 day. Cells were lysed and assayed for luciferase activity. Data are represented as fold induction \pm standard deviation, measured from three independent experiments. ${ }^{*} p$ $<0.01$.

bocytes $^{6}$, we examined the effect of bilobetin on intracellular signaling. When sebocytes were activated by IGF-1, the phosphorylation of IGF-1-R and AKT was significantly increased. Pretreatment with bilobetin did not affect the IGF-1-induced phosphorylation of IGF-1-R, while the phosphorylation of AKT was significantly inhibited by bilobetin. By contrast, phosphorylation of ERK $1 / 2$ induced by IGF-1 was not inhibited by bilobetin (Fig. 3B). These re- sults suggest that action mechanism of bilobetin is primarily related to its inhibitory potential on AKT signaling.

\section{DISCUSSION}

Acne is a very prevalent skin disease, about $85 \%$ of young adults aged 12 to 25 years are affected by this skin prob$\mathrm{lem}^{13}$. Excessive sebum production and increased sebo- 
A

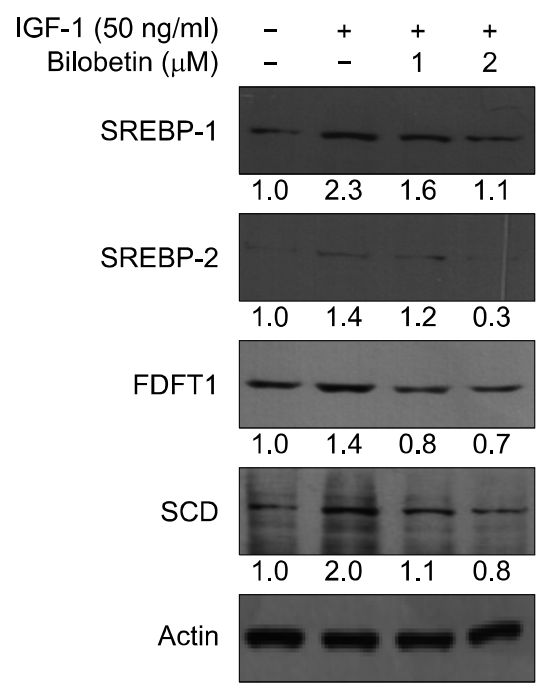

B

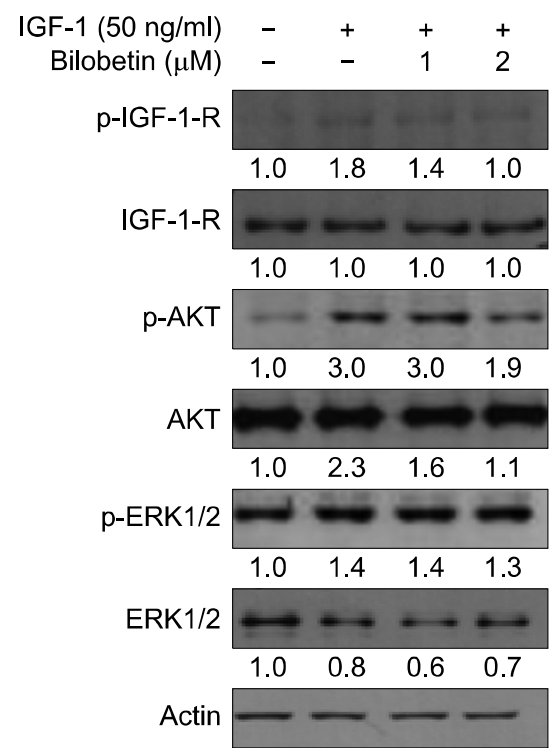

Fig. 3. (A) Effect of bilobetin on the protein level for lipogenic regulators. Simian virus $40 \mathrm{~T}$ (SV40T)-transformed sebocytes were treated with insulin-like growth factor-1 (IGF-1) and bilobetin at the indicated concentrations for 1 day. Protein level for lipogenic regulators was evaluated by Western blot. (B) Effect of bilobetin on intracellular signaling. Cells were pretreated with bilobetin at the indicated concentrations for 30 minutes, and then IGF-1 was treated for 30 minutes. Cellular proteins were prepared and phosphorylation of signaling molecules was evaluated by Western blot. Actin was used as a loading control. Western bands were densitometrically analyzed and normalized with actin. SREBP: sterol response element binding protein, FDFT1: farnesyl-diphosphate farnesyltransferase 1, SCD: stearoyl-CoA desaturase.

cyte proliferation play pivotal roles in the pathogenesis of acne $^{14}$. In non-pathological condition, sebum constitutes the majority of skin surface lipids and exerts its role as a biological barrier by producing anti-microbial activity as well as a physico-chemical barrier of the skin ${ }^{15}$. However, excessive sebum makes the microenvironment surrounding hair follicle more favorable to colonization of lipophilic bacteria such as $P$. acne, thereby contributing to establishment of pathologic condition. In addition to the role for supporting bacterial growth, sebum may exert its role as a direct inducer for acne symptom. For example, squalene monohydroperoxide is the primary peroxidation product in human skin surface lipids that can induce hyperplasia and hyperkeratosis of the epithelium in follicular infundibulum ${ }^{16}$. Thus, targeting the sebum production in sebocytes is a good strategy for preventing acne. In this study, we demonstrated that bilobetin inhibited IGF-1-induced sebum production via the inhibition of AKT signaling in cultured human sebocytes.

Bilobetin is one of biflavonoids isolated from crude leaf extracts of ginkgo biloba ${ }^{8}$. Many biological activities of bilobetin have been reported. For example, bilobetin inhibited cAMP-phosphodiesterase in rat adipose tissue ${ }^{17}$. In other example, bilobetin ameliorates insulin resistance by protein kinase A-mediated phosphorylation of proliferator- activated receptor- $\alpha$ in rats fed a high-fat $\operatorname{diet}^{18}$. In this study, we demonstrated that bilobetin inhibited IGF-1-induced sebum production in sebocytes. Bilobetin significantly inhibited IGF-1-induced increase of lipogenic regulators including SREBP-1 and SREBP-2. Furthermore, bilobetin significantly blocked IGF-1-induced activation of AKT signaling. Since it has been demonstrated that IGF-1 signaling play an important role in sebum production through induction of lipogenic transcription factors such as SREBP $-1^{6}$, our results suggest that effects of bilobetin on sebum production may be due to its inhibitory potential on IGF-1-induced AKT signaling. As previously recognized, IGF-1 promotes seborrhea and inflammation, thereby playing an important role in the pathogenesis of acne $\mathrm{e}^{2,4}$. Therefore, efficient blockage of this signaling is a good strategy in the treatment of acne, and bilobetin can be a candidate for this purpose. Nonetheless, IGF-1 is one factor affecting the sebum production, and there are still many important factors critically involved in pathogenesis of acne, such as androgen and commensals. Thus, the limitation of bilobetin should be considered in the context of developing the drugs for acne treatment.

In summary, we demonstrated that bilobetin inhibited IGF-1induced sebum production in sebocytes through the inhibition of IGF-1-induced AKT signaling. Our results sug- 
gest that bilobetin can be applicable for acne treatment.

\section{ACKNOWLEDGMENT}

This study was supported by a grant of the Korean Health Technology R\&D Project, Ministry of Health \& Welfare, Republic of Korea (Grant No. HN14C0095).

\section{CONFLICTS OF INTEREST}

The authors have nothing to disclose.

\section{ORCID}

Cong Wang, https://orcid.org/0000-0001-7083-2767 Yul-Lye Hwang, https://orcid.org/0000-0002-7595-447X

Xue Mei Li, https://orcid.org/0000-0002-1231-2891

Soo Jung Kim, https://orcid.org/0000-0002-5140-1432

Ming Ji Zhu, https://orcid.org/0000-0003-0835-2548

Jeung-Hoon Lee, https://orcid.org/0000-0002-4869-940X

Ri-Hua Jiang, https://orcid.org/0000-0002-7957-9427

Chang Deok Kim, https://orcid.org/0000-0001-9341-6491

\section{REFERENCES}

1. Zouboulis CC, Picardo M, Ju Q, Kurokawa I, Törőcsik D, Bíró $\mathrm{T}$, et al. Beyond acne: current aspects of sebaceous gland biology and function. Rev Endocr Metab Disord 2016; 17:319-334.

2. Shi VY, Leo M, Hassoun L, Chahal DS, Maibach $\mathrm{HI}$, Sivamani RK. Role of sebaceous glands in inflammatory dermatoses. J Am Acad Dermatol 2015;73:856-863.

3. Chen W, Obermayer-Pietsch B, Hong JB, Melnik BC, Yamasaki O, Dessinioti $C$, et al. Acne-associated syndromes: models for better understanding of acne pathogenesis. J Eur Acad Dermatol Venereol 2011;25:637-646.

4. Kim H, Moon SY, Sohn MY, Lee WJ. Insulin-like growth factor-1 increases the expression of inflammatory biomarkers and sebum production in cultured sebocytes. Ann Dermatol 2017;29:20-25.

5. Krautheim A, Gollnick HP. Acne: topical treatment. Clin Dermatol 2004;22:398-407.
6. Smith TM, Gilliland K, Clawson GA, Thiboutot D. IGF-1 induces SREBP-1 expression and lipogenesis in SEB-1 sebocytes via activation of the phosphoinositide 3-kinase/Akt pathway. J Invest Dermatol 2008;128:1286-1293.

7. Hwang YL, Im M, Lee MH, Roh SS, Choi BW, Kim SJ, et al. Inhibitory effect of imperatorin on insulin-like growth factor1-induced sebum production in human sebocytes cultured in vitro. Life Sci 2016;144:49-53.

8. Briançon-Scheid F, Lobstein-Guth A, Anton R. HPLC separation and quantitative determination of biflavones in leaves from Ginkgo biloba. Planta Med 1983;49:204-207.

9. Im M, Kim SY, Sohn KC, Choi DK, Lee Y, Seo YJ, et al. Epigallocatechin-3-gallate suppresses IGF-I-induced lipogenesis and cytokine expression in SZ95 sebocytes. J Invest Dermatol 2012;132:2700-2708.

10. Melnik BC, Schmitz G. Role of insulin, insulin-like growth factor-1, hyperglycaemic food and milk consumption in the pathogenesis of acne vulgaris. Exp Dermatol 2009;18:833841.

11. Horton JD, Goldstein JL, Brown MS. SREBPs: activators of the complete program of cholesterol and fatty acid synthesis in the liver. J Clin Invest 2002;109:1125-1131.

12. Kim HJ, Miyazaki M, Man WC, Ntambi JM. Sterol regulatory element-binding proteins (SREBPs) as regulators of lipid metabolism: polyunsaturated fatty acids oppose cholesterolmediated induction of SREBP-1 maturation. Ann N Y Acad Sci 2002;967:34-42.

13. Lynn DD, Umari T, Dunnick CA, Dellavalle RP. The epidemiology of acne vulgaris in late adolescence. Adolesc Health Med Ther 2016;7:13-25.

14. Zouboulis CC. Acne and sebaceous gland function. Clin Dermatol 2004;22:360-366.

15. Tóth BI, Oláh A, Szöllosi AG, Czifra G, Bíró T. "Sebocytes' makeup": novel mechanisms and concepts in the physiology of the human sebaceous glands. Pflugers Arch 2011; 461:593-606

16. Picardo M, Ottaviani M, Camera E, Mastrofrancesco A. Sebaceous gland lipids. Dermatoendocrinol 2009;1:68-71.

17. Saponara R, Bosisio E. Inhibition of cAMP-phosphodiesterase by biflavones of Ginkgo biloba in rat adipose tissue. J Nat Prod 1998;61:1386-1387.

18. Kou XH, Zhu MF, Chen D, Lu Y, Song HZ, Ye JL, et al. Bilobetin ameliorates insulin resistance by PKA-mediated phosphorylation of PPARa in rats fed a high-fat diet. Br J Pharmacol 2012;165:2692-2706. 